\title{
The HOG MAPK pathway in Candida albicans: more than a osmosensing pathway
}

Elvira Román*, Inês Correia`, Daniel Prieto, Rebeca Alonso and Jesús Pla.

Departamento de Microbiología y Parasitología-IRYCIS, Facultad de Farmacia, Universidad Complutense de Madrid, Plaza de Ramón y Cajal s/n, E-28040 Madrid, Spain.

- Instituto de Investigação e Inovação em Saúde, Universidade do Porto, Rua Alfredo Allen, 208 | 4200-135 Porto, Portugal

*Corresponding author: elvirarg@farm.ucm.es

Phone: +34 913941617

FAX: +34913941745 


\begin{abstract}
In 1993, Brewster and Gustin described the existence of a kinase whose activity was essential for Saccharomyces cerevisiae to grow in environments with high osmolarity. This led to the discovery of the HOG pathway, a MAP kinase (MAPK) pathway that has revealed crucial to respond to wide range of stress conditions frequently encountered by fungi in their common habitats. MAPK signaling is initiated at the plasma membrane, where triggering stimuli lead to a phosphorylation cascade that ultimately activates transcription factors to ensure an appropriate adaptive response. In pathogenic fungi, the HOG pathway gains special significance as it is involved in traits related to pathogenicity; these include biofilm formation, adhesion to surfaces and morphogenetic and epigenetic transitions. It also plays a role in controlling both the pathogen and the commensal state program. Understanding the signals leading to its activation, the elements of the pathways and the targets of the pathway is therefore of primary importance in the design of novel antifungals.
\end{abstract}


Although fungal infections are not as frequent as those derived from bacteria, they remain as an important cause of human diseases. Candida albicans is a pathogenic yeast that normally inhabits the human body and frequently causes life-threatening invasive infections (called candidiasis) that account for $\sim 19 \%$ of the infections in intensive care units. This microorganism behaves as a harmless commensal of the gastrointestinal and vaginal tract of humans in a significant (albeit not precisely determined) percentage of individuals but is able to cause disease once the host defense mechanisms are compromised (Gow et al. 2012; lliev and Leonardi 2017; Leonardi et al. 2018; Netea and Brown 2012; Romani 2011). This ability to proliferate within the human host is the most probable cause for the high incidence of its infections. Factors such as neutropenia, immune disorders, cancer, diabetes, long-term broadspectrum antibacterial treatment and catheters are predisposing factors to candidiasis. Cryptococcus neoformans is a basidiomycete fungus acquired through inhalation from pigeons' excreta. This yeast may proliferate once inhaled under some conditions; the capsule is an important virulence factor that facilitates blood dissemination and can lead to fatal meningitis. Aspergillus spp. infections are frequently acquired from spores present in the environment; proliferation within immunocompromised individuals may develop in a systemic and fatal disease and are very difficult to control even in specialized hospital units. These three examples illustrate the fact that while most fungi normally cause mild superficial infections, their diseases can be very serious and are far from being controlled in every clinical situation. Effective therapy is much more limited for these infections given the similarity of fungal cells (eukaryotic) with human cells and the development of resistance against certain antifungals that may limit their utility in a near future. New therapeutic options are still necessary and research has therefore focused in the identification of virulence traits as novel therapeutic targets.

MAPK routes are essential sensor mechanisms that transduce environmental inputs to biochemical events that ensure adaptation to the new physiological situation. These routes are conserved among all eukaryotic organisms and control proliferation and stress responses in mammalian cells (Widmann et al. 1999). The central module is composed of three kinases that become sequentially activated by phosphorylation (Kultz 1998). Sensor mechanisms exist at the plasma membrane that connect the stimuli to the MAPK central module, which include G-protein coupled receptors, two-component systems based on histidine-aspartic phosphorylation sensors, integral membrane proteins or tyrosine kinase receptors (Herskowitz, 1995). The signal is eventually transferred to a MAP kinase kinase kinase (MAPKKKs) that phosphorylates a MAP kinase kinase (MAPKKs) which in turn phosphorylates a MAP kinase (MAPKs). The MAPK translocates to the nucleus where it activates different factors that lead to an adaptive transcriptional response.

Activation of these pathways is transient as permanent is frequently prejudicial to the cell; therefore, there are negative and positive feedbacks mechanisms to ensure a timely and regulated response as well as ways to ensure the specificity of the response via scaffold proteins (Saito 2010).

In C. albicans, four MAPK pathways have been described (Monge et al. 2006; Román et al. 2007). The cell integrity (or PKC) pathway regulates the biogenesis of the cell wall, morphogenesis, biofilm formation and virulence. The Cek1mediated pathway, also involved in cell wall formation, was discovered as regulating mating and has been shown to regulate vegetative and invasive growth. Cek2, homologue to ScFus3, is essential for an efficient mating process. We will here focus on the role of the HOG pathway in C. albicans, mediated by the MAPK Hog1 and in particular, in its involvement in important traits which have been grouped in stress, morphogenesis and virulence/commensalism. It must be indicated that, obviously, this is done for clarity in the review. We will, where appropriate, discuss its relation with the cell integrity and CEK1 pathways as they coordinately regulate some traits. We will also highlight its functionality in other fungal pathogens without extensive analyzing their role in other models such as $S$. cerevisiae or Schizosaccharomyces pombe or plant pathogens which have been the subject of excellent reviews (Hohmann 2015; Ikner and Shiozaki 2005; Perez and Cansado 2010). A short section collecting the role of HOG pathway in C. neoformans and certain Aspergillus species is included at the end of the review. 


\section{Stress}

In C. albicans, the HOG (High Osmolarity Glycerol) pathway was first described to mediate adaptation to high osmotic environments. HOG1 was cloned by complementation of $S$. cerevisiae hog1 mutants and its deletion resulted in an increased susceptibility to osmotic stress and a failure to accumulate glycerol, indicating that this metabolite is a compatible solute in C. albicans as in S. cerevisiae (San José et al. 1996). This phenotype was not only observed in hog1 cells but also in pbs2 and ssk2 mutants, being Pbs2 and Ssk2 the MAPKK and the only (in contrast with S.cerevisiae) MAPKKK respectively of the route (Arana et al. 2005; Cheetham et al. 2007). Interestingly, in C. albicans Hog1 is not only activated by osmotic but also by oxidative stress (Alonso-Monge et al. 2003), a somehow unexpected result when discovered although previously suggested from the phenotype observed in S. cerevisiae (Singh 2000). In C. albicans, hog1 mutants are susceptible to a range of oxidants (hydrogen peroxide, $t-\mathrm{BOOH}$ and diamide), heavy metals $\left(\mathrm{Cd}^{2+}\right.$ and $\mathrm{As}^{3+}$ ) and UV light, a phenotype also observed in pbs2 and ssk2 mutants (Alonso-Monge et al. 2003; Cheetham et al. 2007). It has been shown that $\mathrm{H}_{2} \mathrm{O}_{2}$ arrests the $C$. albicans cell cycle and $\mathrm{Hog} 1$ is required to resume the growth after the stress (Correia et al. 2016),

How is the oxidative signal transduced to Hog1 is presently unknown. In S. cerevisiae, the HOG pathway is activated by two different upstream branches (Figure 1). The SLN1-branch relies on a two-component system formed by SLN1, YPD1 and SSK1 genes that activate the redundant Ssk2/Ssk22 MAPKKKs (Posas et al. 1996). The SHO1-branch (Posas and Saito 1997) connects Sho1, a transmembrane protein that interacts with Msb2 and Hkr1 (Cullen et al. 2004; O'Rourke and Herskowitz 2002), Opy2 and Ste50 with Ste20, a PAK-like kinase. Both branches converge at Pbs2 (Posas et al., 2000; de Nadal et al., 2002;Hohmann, 2002). In C. albicans, although both branches exist, only the SLN1-branch is responsible for the activation of Hog1 by oxidative stress, as ssk1 and ssk2 mutants fail to activate Hog1 in response to hydrogen peroxide (Chauhan et al. 2003). This was also confirmed later using ssk2 mutants (Cheetham et al. 2007), indicating that there is no redundancy at the Ssk2 level in C. albicans. The C. albicans SHO1-branch is not involved in the activation of Hog1 but rather in the activation of Cek1 (Román et al. 2005), the homolog of the S. cerevisiae KSS1. In fact, Msb2, a mucin-like protein, does not mediate activation of Hog1 but Cek1 and rather controls the formation of the cell wall (Román et al. 2009). Interestingly, it has been shown that deletion of upstream components of this branch in combination with SSK1 does not impair activation of Hog1 or glycerol accumulation in response to osmotic stress, although the cells remain sensitive to high osmolarity (Román et al., 2005;2009b;Herrero de et al., 2013). This suggests the existence of a third signaling branch that mediates Hog1 activation upon osmotic stress (Cheetham et al. 2011), as occurs with the response to arsenite.

In C. albicans, the transcription factor Sko1 and not Cap1 (Zhang et al. 2000) (the homolog of S. cerevisiae Yap1 (Moye-Rowley et al. 1989)) is responsible, at least partially, for this oxidant specific response (Alonso-Monge et al. 2010). This was inferred from the different oxidant phenotype of hog1 and cap1 mutants, indicating that each gene controls different subsets of genes and later confirmed upon completion of the $C$. albicans genome by transcriptomic analysis (Enjalbert et al. 2006) where a core of Hog1-stress responsive genes was identified. Sko1 regulates the response to osmotic stress in S. cerevisiae (Proft et al. 2001) but also controls a set of oxidative responsive genes (Rep et al. 2001). In C. albicans, sko1 mutants increase the sensitivity of hog1 mutants to oxidants (Alonso-Monge et al. 2010) and also to the mielomonocytic cell line HL-60, whose killing mechanisms occur via generation of oxidative stress. In addition, a genome wide transcriptional analysis revealed different expression of specific oxidative stress response genes, indicating that Sko1 is responsible, at least partially, of this behavior. Interestingly, Hog1 also becomes phosphorylated in response to arsenate (Urrialde et al. 2015) in an Ssk1-dependent process, similarly to what occurs in S. pombe (Rodriguez-Gabriel and Russell 2005). Both arsenate and arsenite increase reactive oxidant species in C. albicans, linking arsenic ion metabolism with the HOG pathway (Urrialde et al. 2017). Recently, it has been reported that the oxidation of Hog1 in a specific Cys residue mediates the response to nitrosative stress (Herrerode-Dios et al. 2018). This suggests that alternative posttranslational modification (not only phosphorylation) regulate the stress-specific outputs of MAPK pathways. 


\section{Morphogenesis and cell wall biogenesis}

Morphogenesis is an essential trait related to virulence in fungi (Rooney and Klein 2002). C. albicans is a dimorphic fungus, being able to switch from a unicellular yeast-like form to a hyphal form in response to stimuli frequently encountered in the human host, where this conversion is favored. Nutritional signals are important for filamentation, as nitrogen deprivation, oxygen availability, serum or $\mathrm{pH}$ strongly influence this response. Filamentation is therefore a very complex and environmentally triggered program where several transcription factors and stimuli have been involved (Liu 2001).

The HOG pathway plays a repressive role on filamentation in C. albicans. hog1, pbs2 or ssk2 display enhanced hyphal formation as shown by their increased ability to form filaments under not fully inducing conditions such as reduced concentrations of serum (Alonso-Monge et al. 1999; Arana et al. 2005; Cheetham et al. 2007) or their appearance on solid media with limiting nitrogen concentration (Eisman et al. 2006). These mutants show a crosstalk between the HOG and the Cek1 mediated pathways rendering in a hyperphosphorylation of Cek1 MAPK which regulates filamentation. In contrast, ssk1 mutants show a reduced ability to filament even in the presence of serum, a phenotype not suppressed by the overexpression of HOG1 (Calera et al. 2000). Interestingly, hog1 mutants (as well as pbs2 and ssk2) express the GATA transcription factor Brg1, a promoter of hyphal formation (Su et al. 2013). As Brg1 is a target of the nutrient sensing Tor1-mediated pathway, this supports the idea that diminishing Tor1 activity via an altered metabolic state nutrition leads to a decrease in Hog1 signaling (by activation of the Ptp2 and Ptp3 phosphatases) that ultimately lead to Brg1 expression.

In C. albicans, the HOG pathway is also involved in the formation of chlamydospores. These structures are frequently considered (but not yet proven) to be forms of resistance, able to survive under harsh environmental conditions (Staib and Morschhauser 2007). As chlamydospores are not usually observed in vivo (Cole et al. 1991) and their proportion is scarce (chlamydospores are usually formed at the tip of the growing hypha), their study has been hampered. Hog1 is; essential for chlamydospore formation (Alonso-Monge et al. 2003) by a mechanism that is independent of the Efg1 regulator, a key regulatory gene involved in this developmental process (Sonneborn et al. 1999). Both efg1 and hog1 are unable to form chlamydospores but overexpression of EFG1 gene in a hog1 mutant -and vice versa- did not restore the phenotype. The reasons for the involvement of Hog1 in this morphogenetic process are presently unclear. One possibility could be the involvement of $\mathrm{Nrg} 1$ (a repressor of hyphal formation (Murad et al. 2001)) that could be regulated by Hog1, as Nrg1 is also involved in chlamydospore formation (Staib and Morschhauser 2005). Another explanation could invoke the fact that these structures are formed under low oxygen concentrations and absence of light, suggesting a role for oxidative stress in this process. Recently, a role for mitochondrial complex I has been demonstrated in gastrointestinal commensalism (Huang et al. 2017). The authors also demonstrate that mannitoldependent morphogenesis is controlled by an oxidative stress signaling pathway that requires Hog1 and Brg1 repression.

The HOG pathway is also involved in cell wall biogenesis. Deletion of hog1 confers a resistance to certain compounds such as nikkomycin Z (an inhibitor of chitin synthesis) other drugs such as Congo red or calcofluor white that interfere with the correct assembly of the cell wall (Alonso-Monge et al. 1999; Román et al. 2005), phenotypes that are not completely shared by pbs2 mutants (Arana et al. 2005). It has been proposed, based on transcriptional studies, that the HOG pathway regulates chitin synthesis coordinately with other pathways (Munro et al., 2007). This could involve other MAP kinases, such as Cek1, which has been also shown to be important in cell wall formation (Eisman et al. 2006; Navarro-García et al. 2005). Osmotic stress can be triggered by an increase in external osmolites but also through the action of killer toxins; interestingly, the $C$. albicans Hog1 has been implicated in the survival to killer toxins from Debaryomycess hansenii (Morales-Menchen et al. 2018), pointing towards a role for this pathway in the construction of the cell wall. 


\section{Commensalism and Pathogenesis}

Since the HOG pathway plays so many crucial roles in the biology of $C$. albicans, it is not surprising the relevance of this pathway in fungal pathogenesis. In fact, although hog1 mutants are hyperfilamentous (therefore, expecting to cause increased tissue damage), they show an attenuated virulence in a mouse model of systemic infection (AlonsoMonge et al., 1999). This can be partially explained by their sensitivity to oxidative and nitrosative stress, even more pronounced in pbs2 strains (Arana et al. 2005), which could result in sensitivity to the phagocytic attack by macrophages and neutrophils that generate reactive oxygen species (ROS) or reactive nitrogen species (RNS) to allow fungal clearance (Arana et al. 2007). A similar behavior is observed with other mutants of the pathway, indicating that the HOG route is important as a therapeutic target.

Recently Hog1 (and also Pbs2) have been also shown to play a critical role in the establishment of $C$. albicans in the mouse gut (colonization). hog1 mutants are unable to colonize the mouse gut even in animals which have a strong reduction of bacterial microbiota by means of antibiotic treatment, indicating that the HOG pathway controls traits essential for the commensalism program (Prieto et al. 2014). The reasons for this behavior are presently unknown, especially as hog1 mutants are sensitive to bile salts and also show a decreased adhesion to mucosa. However, it has been recently described that $C$. albicans cells are able to undergo a morphogenetic transition (called GUT) that makes cells especially prepared to grow in the mammalian tract (Pande et al. 2013). This transition is morphologically and genetically different from the already described white opaque transition and results in cells with enhanced metabolic abilities (Ene et al. 2016; Pande et al. 2013). The conversion from white to GUT cells is determined by Wor1, a master regulator of the white opaque transition, the mating competent phase of this pathogen (Huang et al. 2006; Srikantha et al. 2006) with Wor1-overproducing cells showing increased fitness in the mouse gut. It has been described that HOG1 represses mating in C. albicans (Liang et al. 2014) as hog1 a/a and $\alpha / \alpha$ white cells are efficiently converted to opaque cells. Given the role of the $1 / \alpha 2$ heterodimer as repressor of Wor 1 production, it is tempting to speculate that the HOG pathway could control GUT production via Wor1 or a yet unidentified element. Recently, in C. glabrata Hog1 has been shown to be responsible for lactic acid resistance and revealed essential for fungal cells to compete with Lactobacillus, a common member of endogenous microbiota (Beyer et al. 2018). Whether this could be occurring in the gastrointestinal tract for $C$. albicans is presently unknown.

\section{Other fungi}

The role of the HOG pathway in other yeasts shows similarities as well as distinctive features. In Cryptococcus neoformans, four serotypes are observed based on capsule composition: $A$ (var. grubii), B and C (var. gattii) and D (var. neoformans). Most of the clinical isolates are of type $A$, the most pathogenic which are found to be more resistant to osmotic stress implying that the HOG pathway is involved (Cruz 2000). HOG1 plays different roles depending on the serotype of the clinical strain. In $\mathrm{H} 99$ strain (a type A reference strain), HOG1 represses capsule production, the most important virulence factor that enable colonization of the central nervous system CNS (Bahn et al. 2005). In addition, it also represses the production of melanin, a pigment accumulated within cells in response to specific environmental conditions which is also involved in oxidative stress resistance. By contrast, this is not observed in the less virulent laboratory type D strain JEC21, indicating how evolution has shaped this pathway for host adaptation. Not surprisingly, HOG1 controls filament formation and hog1 and pbs2 mutants show reduced virulence in animal models of cryptococcosis (Bahn et al. 2005). Interestingly, hog1 mutants show also enhanced ability to mate, a phenotype that resembles the situation already indicated for $C$. albicans. It has been recently hypothesized that the opposite role that the PKA and HOG signaling pathways play in response to glucose availability is crucial for the adaptation within the host in either glucose deficient (lungs) or not (CNS) environments (Banerjee et al. 2016).

In Aspergillus nidulans, the HOG pathway is activated in response to high osmolarity exclusively by the SLN1-branch, and the existence of a proline-rich motif in the Pbs2 homolog allows receiving the input from the Sho1-branch (Furukawa et al. 2005). A. fumigatus share many of the elements of the HOG pathway. sakA and pbs2 mutants (lacking the MAPK and MAPKKs respectively) show enhanced sensitivity to osmotic and oxidative stress, as well as to 
amphotericin $B$ and itraconazole antifungals which induce oxidative stress. However, it seems that sakA plays a key role in the physiology of $A$. fumigatus since it also regulates growth and conidia germination (reviewed in (Ma and $\mathrm{Li}$ 2013)). Deletion of the group III histidine kinase NikA showed morphological defects (low conidia and abnormal hyphae), sensitivity to high osmolarity and resistance to fungicides such as fluodioxonil, which have been described to activate the HOG pathway (Hagiwara et al. 2013).

\section{Conclusions}

While the HOG pathway was initially considered to be an osmotic responsive route responsible for allowing environmental yeasts (such as those on grapes' surfaces) to survive in water limitations periods, it is evident that this is not the case. Rather, the HOG is involved (and evolved) in human pathogenic fungi (not only C. albicans) to coordinately regulate traits such as cell wall biogenesis, morphogenesis and resistance to stress which have profound implications in their adaptation to the host. The existence of drugs which target this pathway combined with the availability to develop efficient and robust chemical and genetic screenings will enable to identify new drugs that may contribute to the control of human fungal diseases.

\section{Acknowledgments}

Work in our laboratory is supported by the Spanish Ministerio de Economía y Competitividad under Grant BIO201564777-P and by Comunidad de Madrid under InGEMICS-CM S2017/BMD3691 (Group COMIPAT).

\section{References}

Alonso-Monge R et al. (1999) Role of the mitogen-activated protein kinase Hog1p in morphogenesis and virulence of Candida albicans J Bacteriol 181:3058-3068

Alonso-Monge R, Navarro-Garcia F, Roman E, Negredo Al, Eisman B, Nombela C, Pla J (2003) The Hog1 mitogenactivated protein kinase is essential in the oxidative stress response and chlamydospore formation in Candida albicans Eukaryot Cell 2:351-361

Alonso-Monge R, Román E, Arana DM, Prieto AD, Urrialde V, Nombela C, Pla J (2010) The Sko1 protein represses the yeast-to-hypha transition and regulates the oxidative stress response in Candida albicans Fungal Genet Biol 47:587-601

Arana DM, Alonso-Monge R, Du C, Calderone R, Pla J (2007) Differential susceptibility of mitogen-activated protein kinase pathway mutants to oxidative-mediated killing by phagocytes in the fungal pathogen Candida albicans Cell Microbiol 9:1647-1659 doi:10.1111/j.1462-5822.2007.00898.x

Arana DM, Nombela C, Alonso-Monge R, Pla J (2005) The Pbs2 MAP kinase kinase is essential for the oxidativestress response in the fungal pathogen Candida albicans Microbiology 151:1033-1049 doi:10.1099/mic.0.27723-0

Bahn YS, Kojima K, Cox GM, Heitman J (2005) Specialization of the HOG pathway and its impact on differentiation and virulence of Cryptococcus neoformans Mol Biol Cell 16:2285-2300 doi:10.1091/mbc.e04-11-0987

Banerjee D, Bloom AL, Panepinto JC (2016) Opposing PKA and Hog1 signals control the post-transcriptional response to glucose availability in Cryptococcus neoformans Mol Microbiol 102:306-320 doi:10.1111/mmi.13461

Beyer R et al. (2018) Competition of Candida glabrata against Lactobacillus is Hog1 dependent Cell Microbiol 20:e12943 doi:10.1111/cmi.12943

Calera JA, Zhao XJ, Calderone R (2000) Defective hyphal development and avirulence caused by a deletion of the SSK1 response regulator gene in Candida albicans Infect Immun 68:518-525

Chauhan N, Inglis D, Román E, Pla J, Li D, Calera JA, Calderone R (2003) Candida albicans response regulator gene SSK1 regulates a subset of genes whose functions are associated with cell wall biosynthesis and adaptation to oxidative stress Eukaryot Cell 2:1018-1024 
Cheetham J et al. (2011) MAPKKK-independent regulation of the Hog1 stress-activated protein kinase in Candida albicans J Biol Chem 286:42002-42016 doi:10.1074/jbc.M111.265231

Cheetham J, Smith DA, da Silva DA, Doris KS, Patterson MJ, Bruce CR, Quinn J (2007) A single MAPKKK regulates the Hog1 MAPK pathway in the pathogenic fungus Candida albicans Mol Biol Cell 18:4603-4614

Cole GT, Seshan KR, Phaneuf M, Lynn KT (1991) Chlamydospore-like cells of Candida albicans in the gastrointestinal tract of infected, immunocompromised mice Canadian Journal of Microbiology 37:637-646

Correia I, Alonso-Monge R, Pla J (2016) The Hog1 MAP Kinase Promotes the Recovery from Cell Cycle Arrest Induced by Hydrogen Peroxide in Candida albicans Front Microbiol 7:2133 doi:10.3389/fmicb.2016.02133

Cullen PJ et al. (2004) A signaling mucin at the head of the Cdc42- and MAPK-dependent filamentous growth pathway in yeast Genes Dev 18:1695-1708 doi:10.1101/gad.1178604

Eisman B, Alonso-Monge R, Román E, Arana DM, Nombela C, Pla J (2006) The Cek1 and Hog1 mitogen-activated protein kinases play complementary roles in cell wall biogenesis and chlamydospore formation in the fungal pathogen Candida albicans Eukaryot Cell 5:347-358

Ene IV, Lohse MB, Vladu AV, Morschhauser J, Johnson AD, Bennett RJ (2016) Phenotypic Profiling Reveals that Candida albicans Opaque Cells Represent a Metabolically Specialized Cell State Compared to Default White Cells MBio 7 doi:10.1128/mBio.01269-16

Enjalbert B, Smith DA, Cornell MJ, Alam I, Nicholls S, Brown AJ, Quinn J (2006) Role of the Hog1 stress-activated protein kinase in the global transcriptional response to stress in the fungal pathogen Candida albicans Mol Biol Cell 17:1018-1032

Furukawa K, Hoshi Y, Maeda T, Nakajima T, Abe K (2005) Aspergillus nidulans HOG pathway is activated only by twocomponent signalling pathway in response to osmotic stress Mol Microbiol 56:1246-1261

Gow NA, van de Veerdonk FL, Brown AJ, Netea MG (2012) Candida albicans morphogenesis and host defence: discriminating invasion from colonization Nature Reviews in Microbiology 10:112-122 doi:10.1038/nrmicro2711

Hagiwara D et al. (2013) NikA/TcsC histidine kinase is involved in conidiation, hyphal morphology, and responses to osmotic stress and antifungal chemicals in Aspergillus fumigatus PLoS One 8:e80881 doi:10.1371/journal.pone.0080881

Herrero-de-Dios C et al. (2018) Redox Regulation, Rather than Stress-Induced Phosphorylation, of a Hog1 MitogenActivated Protein Kinase Modulates Its Nitrosative-Stress-Specific Outputs MBio 9 doi:10.1128/mBio.0222917

Hohmann S (2015) An integrated view on a eukaryotic osmoregulation system Curr Genet 61:373-382 doi:10.1007/s00294-015-0475-0

Huang G, Wang H, Chou S, Nie X, Chen J, Liu H (2006) Bistable expression of WOR1, a master regulator of whiteopaque switching in Candida albicans Proceedings of the National Academy of Sciences of the United States of America 103:12813-12818

Huang X et al. (2017) Mitochondrial complex I bridges a connection between regulation of carbon flexibility and gastrointestinal commensalism in the human fungal pathogen Candida albicans PLoS Pathog 13:e1006414 doi:10.1371/journal.ppat.1006414

Ikner A, Shiozaki K (2005) Yeast signaling pathways in the oxidative stress response Mutat Res 569:13-27

Iliev ID, Leonardi I (2017) Fungal dysbiosis: immunity and interactions at mucosal barriers Nat Rev Immunol doi:10.1038/nri.2017.55

Kultz D (1998) Phylogenetic and functional classification of mitogen- and stress-activated protein kinases J MolEvol 46:571-588

Leonardi I et al. (2018) CX3CR1(+) mononuclear phagocytes control immunity to intestinal fungi Science 359:232-236 doi:10.1126/science.aao1503

Liang SH, Cheng JH, Deng FS, Tsai PA, Lin CH (2014) A novel function for Hog1 stress-activated protein kinase in controlling white-opaque switching and mating in Candida albicans Eukaryot Cell 13:1557-1566 doi:10.1128/ec.00235-14

Liu H (2001) Transcriptional control of dimorphism in Candida albicans Curr Opin Microbiol 4:728-735

Ma D, Li R (2013) Current understanding of HOG-MAPK pathway in Aspergillus fumigatus Mycopathologia 175:13-23 doi:10.1007/s11046-012-9600-5

Monge RA, Roman E, Nombela C, Pla J (2006) The MAP kinase signal transduction network in Candida albicans Microbiology 152:905-912 doi:10.1099/mic.0.28616-0 
Morales-Menchen A et al. (2018) Non-canonical Activities of Hog1 Control Sensitivity of Candida albicans to Killer Toxins From Debaryomyces hansenii Front Cell Infect Microbiol 8:135 doi:10.3389/fcimb.2018.00135

Moye-Rowley WS, Harshman KD, Parker CS (1989) Yeast YAP1 encodes a novel form of the jun family of transcriptional activator proteins Genes and Development 3:283-292

Murad AM et al. (2001) NRG1 represses yeast-hypha morphogenesis and hypha-specific gene expression in Candida albicans EMBO J 20:4742-4752

Navarro-García F, Eisman B, Fiuza SM, Nombela C, Pla J (2005) The MAP kinase Mkc1p is activated under different stress conditions in Candida albicans Microbiology 151:2737-2749

Netea MG, Brown GD (2012) Fungal infections: the next challenge Curr Opin Microbiol 15:403-405 doi:10.1016/j.mib.2012.07.002

O'Rourke SM, Herskowitz I (2002) A third osmosensing branch in Saccharomyces cerevisiae requires the Msb2 protein and functions in parallel with the Sho1 branch Mollecular and Cellular Biology 22:4739-4749

Pande K, Chen C, Noble SM (2013) Passage through the mammalian gut triggers a phenotypic switch that promotes Candida albicans commensalism Nat Genet 45:1088-1091

Perez P, Cansado J (2010) Cell integrity signaling and response to stress in fission yeast Current Protein Peptide Science 11:680-692

Posas F, Saito H (1997) Osmotic activation of the HOG MAPK pathway via Ste11p MAPKKK: scaffold role of Pbs2p MAPKK Science 276:1702-1705

Posas F, Wurgler-Murphy SM, Maeda T, Witten EA, Thai TC, Saito H (1996) Yeast HOG1 MAP kinase cascade is regulated by a multistep phosphorelay mechanism in the SLN1-YPD1-SSK1 "two-component" osmosensor Cell 86:865-875

Prieto AD, Román E, Correia I, Pla J (2014) The HOG pathway is critical for the colonization of the mouse gastrointestinal tract by Candida albicans PLoS ONE 9:e87128

Proft M, Pascual-Ahuir A, de Nadal E, Arino J, Serrano R, Posas F (2001) Regulation of the Sko1 transcriptional repressor by the Hog1 MAP kinase in response to osmotic stress EMBO J 20:1123-1133

Rep M, Proft M, Remize F, Tamas M, Serrano R, Thevelein JM, Hohmann S (2001) The Saccharomyces cerevisiae Sko1p transcription factor mediates HOG pathway-dependent osmotic regulation of a set of genes encoding enzymes implicated in protection from oxidative damage Mol Microbiol 40:1067-1083

Rodriguez-Gabriel MA, Russell P (2005) Distinct signaling pathways respond to arsenite and reactive oxygen species in Schizosaccharomyces pombe Eukaryot Cell 4:1396-1402

Román E, Arana DM, Nombela C, Alonso-Monge R, Pla J (2007) MAP kinase pathways as regulators of fungal virulence Trends Microbiol 15:181-190

Román E, Cottier F, Ernst JF, Pla J (2009) Msb2 signaling mucin controls activation of Cek1 mitogen-activated protein kinase in Candida albicans Eukaryot Cell 8:1235-1249

Román E, Nombela C, Pla J (2005) The Sho1 adaptor protein links oxidative stress to morphogenesis and cell wall biosynthesis in the fungal pathogen Candida albicans Mol Cell Biol 25:10611-10627

Romani L (2011) Immunity to fungal infections Nature Reviews in Immunology 11:275-288 doi:10.1038/nri2939

Rooney PJ, Klein BS (2002) Linking fungal morphogenesis with virulence Cell Microbiol 4:127-137

Saito H (2010) Regulation of cross-talk in yeast MAPK signaling pathways Curr Opin Microbiol 13:677-683

San José C, Alonso-Monge R, Pérez-Díaz RM, Pla J, Nombela C (1996) The mitogen-activated protein kinase homolog HOG1 gene controls glycerol accumulation in the pathogenic fungus Candida albicans $\mathrm{J}$ Bacteriol 178:5850-5852

Singh KK (2000) The Saccharomyces cerevisiae SIn1p-Ssk1p two-component system mediates response to oxidative stress and in an oxidant-specific fashion Free RadicBiol Med 29:1043-1050

Sonneborn A, Bockmuhl DP, Ernst JF (1999) Chlamydospore formation in Candida albicans requires the Efg1p morphogenetic regulator Infect Immun 67:5514-5517

Srikantha T et al. (2006) TOS9 regulates white-opaque switching in Candida albicans Eukaryot Cell 5:1674-1687

Staib P, Morschhauser J (2005) Differential expression of the NRG1 repressor controls species-specific regulation of chlamydospore development in Candida albicans and Candida dubliniensis Mol Microbiol 55:637-652

Staib P, Morschhauser J (2007) Chlamydospore formation in Candida albicans and Candida dubliniensis --an enigmatic developmental programme Mycoses 50:1-12

Su C, Lu Y, Liu H (2013) Reduced TOR signaling sustains hyphal development in Candida albicans by lowering Hog1 basal activity Mol Biol Cell 24:385-397 doi:10.1091/mbc.E12-06-0477 
Urrialde V, Alburquerque B, Guirao-Abad JP, Pla J, Argüelles JC, Alonso-Monge R (2017) Arsenic inorganic compounds cause oxidative stress mediated by the transcription factor PHO4 in Candida albicans Microbiol Res 203:10-18 doi:10.1016/j.micres.2017.06.004

Urrialde V, Prieto D, Pla J, Alonso-Monge R (2015) The Pho4 transcription factor mediates the response to arsenate and arsenite in Candida albicans Front Microbiol 6:118 doi:10.3389/fmicb.2015.00118

Widmann C, Gibson S, Jarpe MB, Johnson GL (1999) Mitogen-activated protein kinase: conservation of a three-kinase module from yeast to human Physiol Rev 79:143-180

Zhang X, De Micheli M, Coleman ST, Sanglard D, Moye-Rowley WS (2000) Analysis of the oxidative stress regulation of the Candida albicans transcription factor, Cap1p Mol Microbiol 36:618-629 